\title{
Near-wake measurement in a rotor/stator axial compressor using slanted hot-wire technique
}

\author{
S. T. Hsu, A. M. Wo
}

\begin{abstract}
Three-dimensional near-wake structure behind a rotor was measured using slanted hot-wire technique in a large-scale, low-speed, rotor/stator axial compressor. Unsteady flow interaction between blade rows was varied by setting the axial gap between rows at $10 \%$ and $30 \%$ of rotor chord. Results show that stronger flow interactions between blade rows, or closer axial gap, produce more pronounced time variation within the rotor wake. All parameters measured - three component velocities, yaw and pitch angles - varied strongly within the wake, and are quantified.

\section{List of symbols}

$C \quad$ airfoil chord (same for all blades)

$u_{a}, u_{\theta}, u_{r}$ axial, tangential and radius components of velocity in compressor

\section{Coordinates}

$\mathrm{V}_{b} \quad$ blade velocity at mid-span

$W \quad$ rotor relative velocity
\end{abstract}

\section{1}

\section{Introduction}

Blade wakes in turbomachines are important consideration not only due to their strong link with losses in performance but also their interaction with the downstream blade row can cause aeroelastic concerns. Within an axial compressor almost never will a wake be completely mixed out before encountering the downstream blade; the typical axial gap between blade rows is only $30 \%$ of a chord.

As the axial gap between blade rows is decreased to $10 \%$ chord, for example, the influence of the downstream pressure field can reach the trailing edge of the wake-producing blade. Among others, Fleeter et al. (1981) carried out an experimental study on the effect of axial spacing on gust response for a rotor/stator configuration. Gallus et al. (1982) undertook extensive investigation of the blade number ratio and blade row spacing on stator dynamic loading and stage sound

Received: 8 July 1996/Accepted: 29 May 1997

S. T. Hsu, A. M. Wo

Institute of Applied Mechanics, National Taiwan University,

Taipei 106, Taiwan, Republic of China

Correspondence to: A. M. Wo

This work was supported by the National Science Council of the Republic of China (NSC-85-2212-E-002-040).
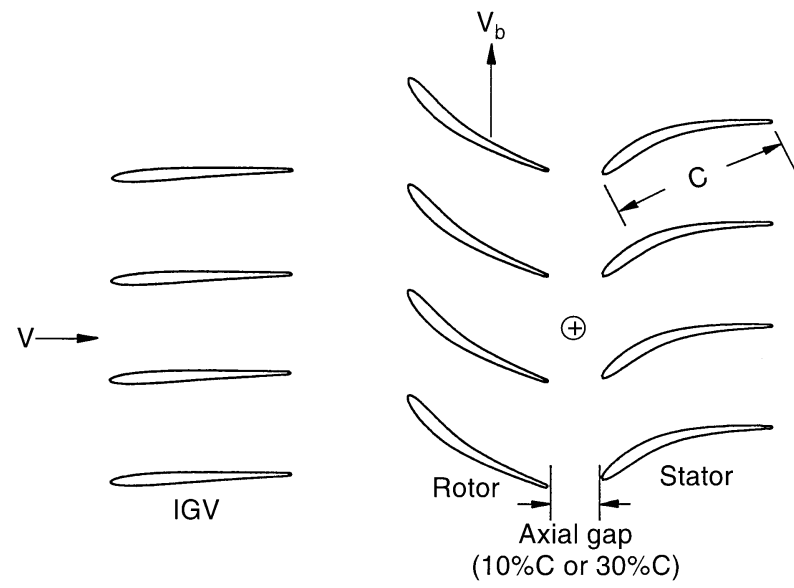

$\oplus$ Slanted hot-wire location to measure rotor wake

Fig. 1. Sketch of the compressor IGV, rotor and stator blades

pressure level. They found that both vortical and potential effects are important in determining the blade response for very small gap.

This work presents some results on the near-wake of a rotor/stator axial compressor, with inlet guide vanes located at $1.75 \mathrm{C}$ upstream of the rotor leading edge to allow for wake dissipation (Fig. 1). The blade row interaction was varied by setting the axial gap at $10 \%$ and $30 \%$ rotor chord, and at two time-mean blade loadings. The compressor achieves staticto-static efficiency of near $90 \%$ for both axial gaps. Threedimensional unsteady wake profile was measured by a single slanted hot-wire with ensemble-averaging. It is not the intention of this work to improve the measurement technique, which has been reported by Schmidt and Okiishi (1977), among others, but to focus on the unsteady wake itself.

\section{2}

\section{Measurement techniques}

Velocity and flow angle measurements in the gap region between two blade rows were made with a Tungsten slanted hot-wire of $5 \mu \mathrm{m}$ diameter (TSI model 1263A-T1.5 and model IFA 100 anemometer). The hot-wire was calibrated in a windtunnel at velocities ranging from 0 to $40 \mathrm{~m} / \mathrm{s}$. Frequency response of $150 \mathrm{kHz}$ was attained using a $1: 1$ bridge. The overall measurement technique was similar to that of Schmidt and Okiishi (1977). Care was exercised to ensure the flow angles were within the calibration range throughout all three orientations, especially within the wake region. 
Sources of error in the measurement technique composed of uncertainties involved in the calibration procedure, positioning of the probe for measurement, ensemble average process, and numerical calculation to resolve the three velocity components. Each source was estimated independently (Hsu 1997). The overall error in the measured velocity within the wake region is estimated to be about $3 \%$.

\section{3}

\section{Results and discussions}

\section{1}

Rotor wake velocities and flow angles ( $10 \%$ chord axial gap)

Figure 2 presents the three-dimensional rotor wake profile, pitch and yaw angles measured at the blade rows mid-gap and stator mid-pitch, for near design and high loadings. The axial velocity profiles, Figs. $2 \mathrm{a}$ and $\mathrm{f}$, show a greater change corresponding to the pressure surface $(t / T=0.55$ to 0.6$)$ than that of the suction surface $(t / T=0.6$ to 0.75$)$, which is due to thinner boundary layer on the pressure surface. Comparison between the two loadings, Figs. $2 \mathrm{a}$ and $\mathrm{f}$, reveal both wake defect and wake width are larger for higher loading, with essentially no variation outside the wake. Note that the slope on both sides of the wake is considered smooth suggesting the wake defect is captured correctly.

The circumferential velocity profiles, Figs. $2 \mathrm{~b}$ and g, show an increase within the wake, which maximizes at the minimum axial velocity point. This clearly illustrates the point made by Kerrebrock and Mikolajczak (1970) that the wake possess an excess of circumferential momentum, which increases with loading.
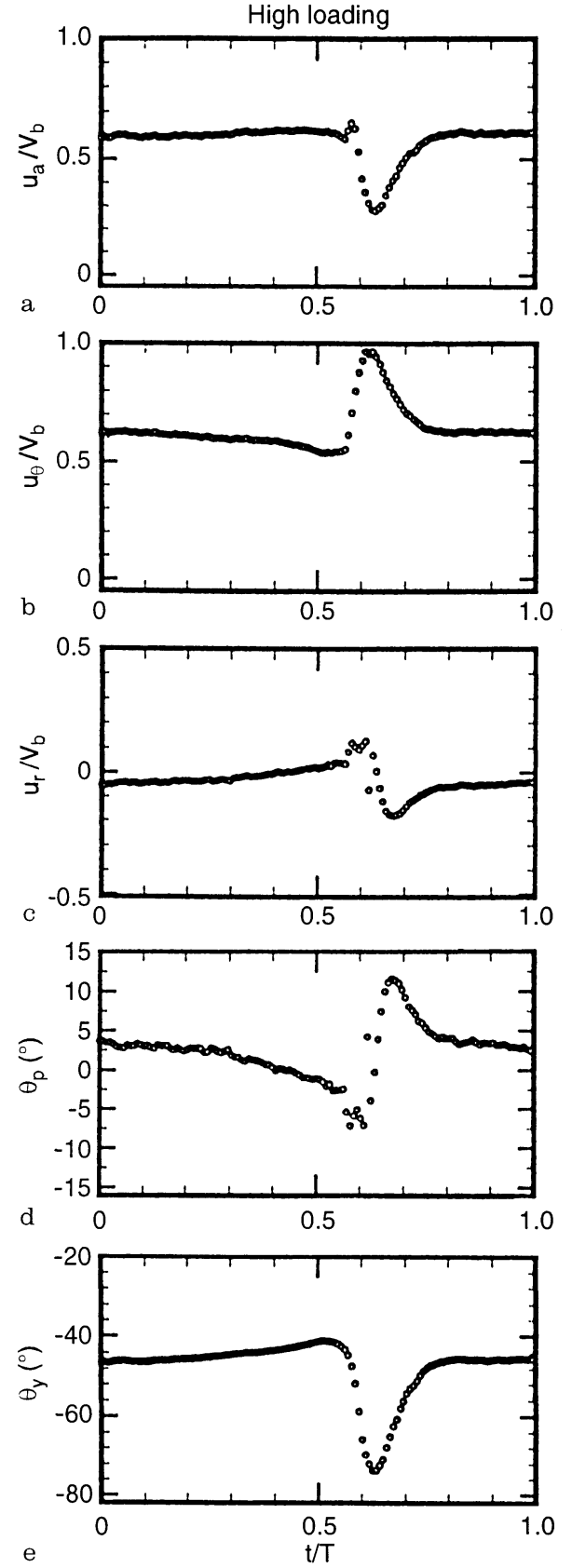
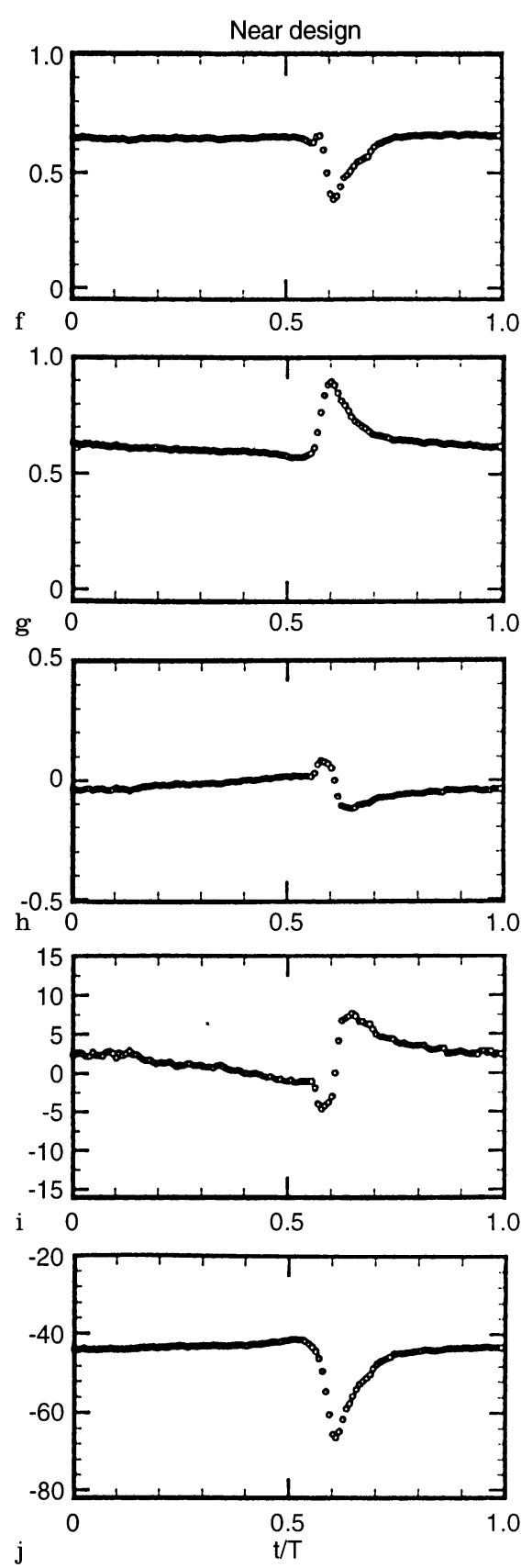

Fig. $2 \mathbf{a}-\mathbf{j}$. Rotor wake properties at the mid-gap and stator mid-pitch position at $10 \%$ chord axial gap between blade rows. $\mathbf{a}, \mathbf{f}$ Axial velocity; $\mathbf{b}, \mathbf{g}$ tangential velocity; $c, h$ radius velocity; $d, i$ pitch angle; $e, j$ yaw angle 
Figures $2 \mathrm{c}$ and $\mathrm{h}$ present the unsteady radial velocities, which are much smaller than either axial or circumferential velocities. The radial velocity is again largest within the wake, while the flow can be considered as two-dimensional outside of the wake region.

Figures $2 \mathrm{~d}$ and i present the variation of flow pitch angle to the hot-wire sensor over an unsteady cycle. Within the compressor, non-zero pitch angle implies presence of radial flow. This can be seen by the similarity with the unsteady radial velocity, except for a sign change. The important point is that the unsteady pitch angle varies from $-7^{\circ}$ to $12^{\circ}$ at high loading, which is within the range of calibration $\left(-15^{\circ}\right.$ to $\left.15^{\circ}\right)$ even for flow in the near-wake region. The flow yaw angle to the sensor, Figs. $2 \mathrm{e}$ and $2 \mathrm{j}$, varies from $-40^{\circ}$ to $-70^{\circ}$, which is also within the calibration range $\left(-80^{\circ}\right.$ to $\left.-8^{\circ}\right)$. One expects the calibration to be in jeopardy for large negative yaw angle and large positive pitch angle, since the resolution deteriorates. This corresponds to the minimum wake velocity point.

Examination of velocities in all three directions at that instant in time reveals no erratic variation.

\section{2}

Rotor wake velocities and flow angles ( $30 \%$ chord axial gap)

Figure 3 presents data similar to that of Fig. 2 except for lower level of unsteady interaction between blade rows $(30 \%$ chord axial gap). With measurements also made at the mid-gap and stator mid-pitch location, which corresponds to $15 \%$ chord behind the rotor trailing edge compared to $5 \%$ prior. Thus the diffusion process occurs over a slightly longer period resulting in a wake with less defect and larger wake width, as shown by
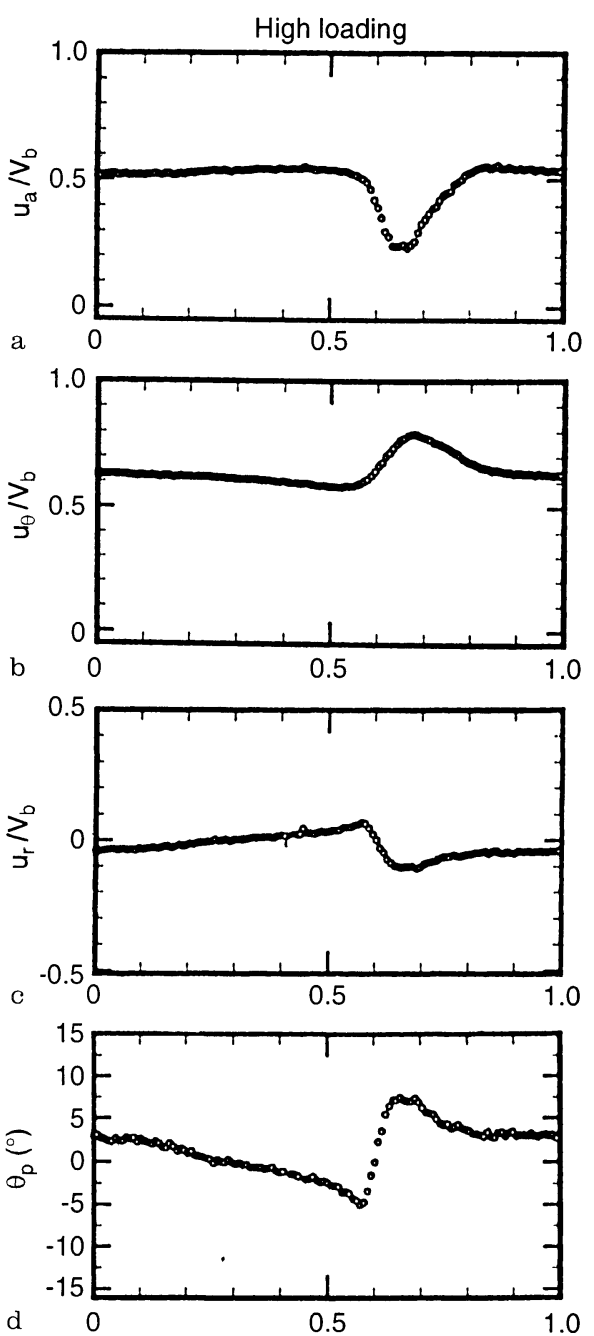

$$
\text { d }
$$

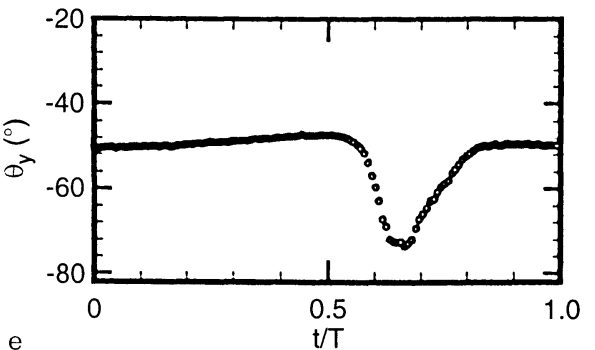

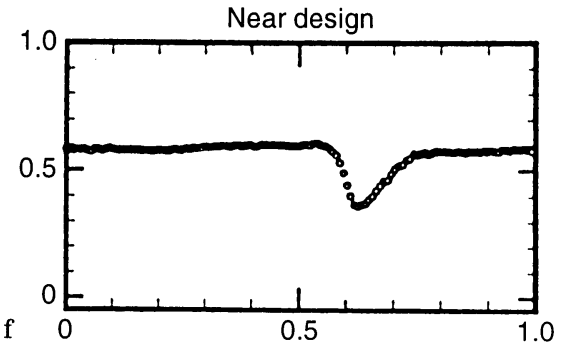
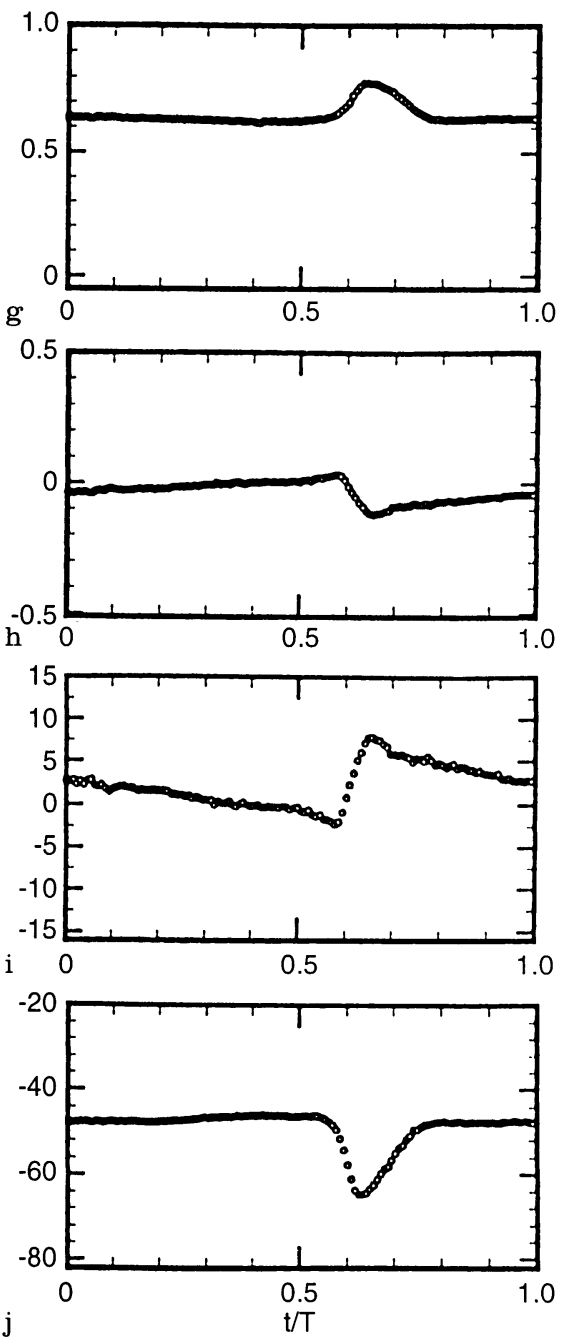

Fig. 3a-j. Rotor wake properties at the mid-gap and stator mid-pitch position at $30 \%$ chord axial gap between blade rows. a, f Axial velocity; $\mathbf{b}, \mathbf{g}$ tangential velocity; $c, h$ radius velocity; $\mathbf{d}, \mathbf{i}$ pitch angle; $\mathbf{e}, \mathbf{j}$ yaw angle 
comparing Fig. 3a with Fig. 2a and Fig. 3f with Fig. 2f. The increase in the minimum wake velocity results in a decrease of circumferential and radial velocities. Thus the unsteady variation in pitch and yaw angles relative to the sensor during the wake region are also less pronounced.

\section{4}

\section{Conclusions}

This paper addresses the unsteady rotor wake, measured by a slanted hot-wire, in an experimental rotor/stator axial compressor, at various levels of flow interactions and loadings. The primary mechanism of blade row interaction is the presence of the downstream stator which causes an unsteady potential disturbance upstream. Results show stronger flow interactions between blade rows, at $10 \%$ chord axial gap, which produce more rapid time-variation in the rotor wake for all variables measured - 3D velocities, and sensor yaw and pitch angles. The radial flow is strongest within the wake, and increases with loading for both axial gap cases. The use of slanted hot-wire measurement technique was shown to be acceptable even at near-wake and at the level of blade row interactions studied. The instantaneous yaw and pitch angles was within calibration range, even within the wake region where large flow angle change was expected.

\section{References}

Fleeter S; Jay RL; Bennett WA (1981) Wake induced time-variant aerodynamics including rotor-stator axial spacing effects. ASME J Fluids Eng 103: 59-66

Gallus HE; Lambertz J; Wallman Th (1982) The influence of blade number ratio and blade row spacing on axial-flow compressor stator blade dynamic load and stage sound pressure level. J Eng for Power 104: 633-641

Hsu ST (1997) Experimental study of vortical and potential interaction in an axial compressor. Doctoral Thesis, Institute of Applied Mechanics, National Taiwan University

Kerrebrock JL; Mikolajczak AA (1970) Intra-stator transport of rotor wakes and its effects on compressor performance. Trans ASME J Eng for Power 92: 359-369

Schmidt DP; Okiishi TH (1977) Multistage axial-flow turbomachine wake production, transport, and interaction. AIAA J 15: 1138-1145 